\title{
Crohn's Disease with Atypical Extra-Intestinal Manifestations Developing Under Treatment with Vedolizumab
}

\author{
Aasem Abu Shtaya ${ }^{1,2}$, Shai Cohen ${ }^{1,2}$, Yana Kogan ${ }^{2,3}$, Michal Shteinberg $^{2,3}$, Ori Sagool $^{2,4}$ \\ ${ }^{1}$ Department of Internal Medicine "B", Lady Davis Carmel Medical Center, Haifa, Israel \\ ${ }^{2}$ Technion - Israel Institute of Technology, The B. Rappaport Faculty of Medicine, Haifa, Israel \\ ${ }^{3}$ Pulmonology Institute and CF center, Lady Davis Carmel Medical Center, Haifa, Israel \\ ${ }^{4}$ Department of Gastroenterology, Lady Davis Carmel Medical Center, Haifa, Israel
}

\section{Doi: 10.12890/2021_002265- European Journal of Case Reports in Internal Medicine - C EFIM 2021}

Received: 03/01/2021

Accepted: 20/01/2021

Published: $19 / 02 / 2021$

\begin{abstract}
How to cite this article: Abu Shtaya A, Cohen C, Kogan Y, Shteinberg M, Sagool O. Crohn's disease with atypical extra-intestinal manifestations developing
\end{abstract} under treatment with vedolizumab. EJCRIM 2021;8: doi:10.12890/2021_002265.

Conflicts of Interests: The Authors declare that there are no competing interests.

This article is licensed under a Commons Attribution Non-Commercial 4.0 License

\section{ABSTRACT}

Crohn's disease is a chronic inflammatory bowel disease that can affect any part of the GI tract, which is frequently associated with extraintestinal manifestations. Pulmonary parenchymal disease is very uncommon and usually considered to be debilitating and harder to diagnose. Pulmonary granulomas are rarely described in the literature as a complication of Crohn's disease. Here, we present a patient with Crohn's disease exacerbation who developed granulomatous lung disease under treatment with vedolizumab. Our case may add evidence to the emerging theory that gut-selective biologic agents could lead to upregulation of some pro-inflammatory factors leading to the evolution of pulmonary disease.

\section{LEARNING POINTS}

- Pulmonary parenchymal diseases are rare in Crohn's disease but they can be debilitating and life-threatening as they are usually tardily diagnosed; awareness of this association is of high value and could potentially shorten the time to a definite diagnosis.

- Pulmonary manifestations of Crohn's disease could be subclinical without any respiratory complaints and not diagnosed with conventional imaging modalities such as chest $x$-ray.

- Gut-selective biologic agents could lead to the emergence of extra-intestinal manifestations due to upregulation of multiple proinflammatory cytokines.

\section{KEYWORDS}

Crohn's disease, granuloma, vedolizumab, infliximab

\section{CASE DESCRIPTION}

This case involves a 31-year-old male patient, diagnosed with small intestinal Crohn's disease at the age of 18, who had previously been treated with adalimumab, 6-mercaptopurine and 5-aminosalicylic acid with a partial response to the 3 treatments and frequent relapses requiring treatment with systemic steroids. He suffered from severe anaemia and hypoalbuminaemia secondary to his illness and had severe osteoporosis with a T-score of -4.5 due to frequent steroid use.

He was admitted to our department due to a febrile illness, abdominal pain and bloody diarrhoea that started 3 weeks prior to admission. Physical examination was significant for cachexia and sarcopenia; the patient weighed $60 \mathrm{~kg}$ at admission (BMI=23). Abdominal examination revealed diffuse tenderness. Testing for inflammatory biomarkers and stool calprotectin, along with stool samples for culture, parasites 
and Clostridium toxin were negative, while stool multiplex PCR assessment for an infectious process was also negative. A chest x-ray on admission was normal (Fig. 1) and abdominal CT enterography demonstrated minimal fat stranding. Upper endoscopy revealed mild gastritis. Colonoscopy, as well as biopsies along the colon and the terminal ileum were normal. With a working hypothesis of Crohn's disease exacerbation enteral feeding was withheld and the patient was treated with ceftriaxone, metronidazole and azithromycin, without response. We did not initiate steroidal therapy at that stage due to his severe osteoporosis.

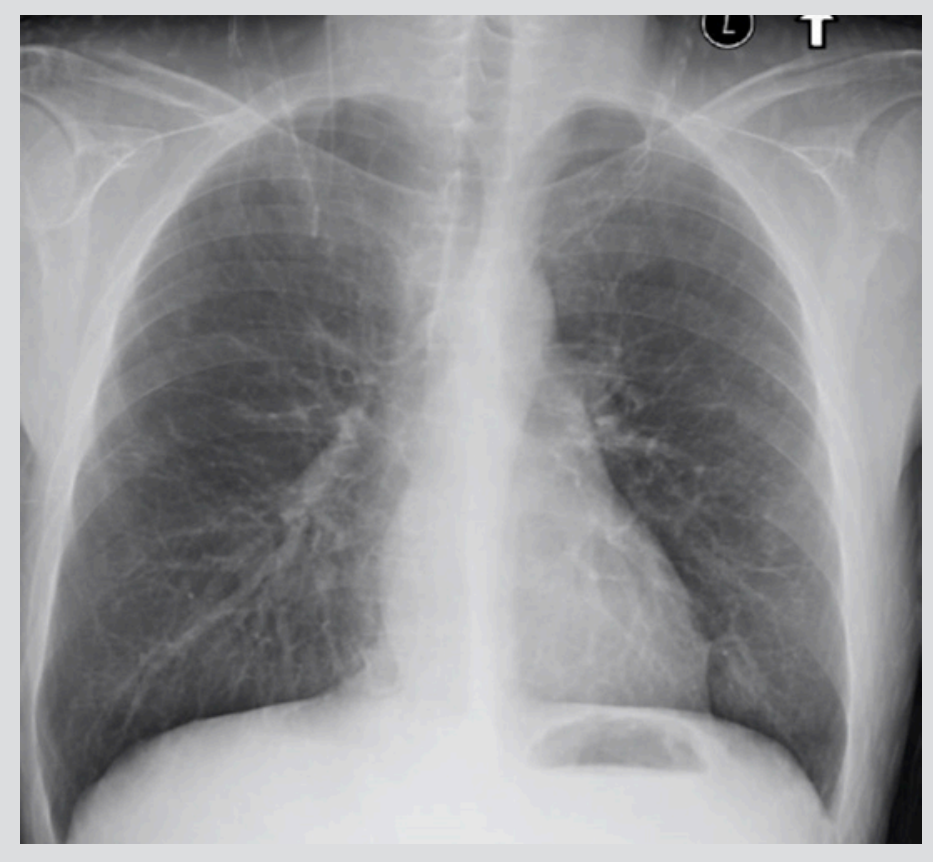

Figure 1. Evolution of pleural effusion, at day 10 (A, with iatrogenic pneumothorax) and 1 month later (B)

In order to better evaluate his intestinal disease, the patient underwent magnetic resonance enterography, which did not yield any significant findings.

After 10 days of antibiotic therapy the febrile illness continued, along with severe diarrhoea, weight loss and a deteriorating general medical condition. Antibiotic therapy was stopped, total parenteral nutrition was initiated and the patient received a single loading dose of gutselective vedolizumab. A few days later the patient reported improvement of abdominal pain and the frequency of diarrhoea, but his febrile illness persisted. We intensified the investigations, including repeat blood and urine cultures, viral and immunological serology testing, cryoglobulin measurement, echocardiography, ophthalmological evaluation, chest x-ray, abdominal x-ray and ultrasonography. All tests returned normal. Due to further deterioration the patient received a course of steroidal therapy without any improvement.

After being treated with antibiotics, vedolizumab and steroids, fever continued on a daily basis. Hence, the patient underwent PET-CT that demonstrated increased metabolic activity in both lungs without any enhancement along the GI tract (Fig. 2), while a repeat chest x-ray (that was normal a few days previously) revealed new bilateral infiltrates on both lungs and a new left pleural effusion (Fig. 3). Thoracentesis was performed revealing an exudative effusion; the effusion tested negative for an infectious process through multiplex PCR panels and cytology assessment was also negative.

A bronchoscopy with bronchoalveolar lavage revealed no endobronchial abnormality; the lavage fluid was also negative for infectious causes (including COVID-19 PCR testing). A transbronchial biopsy yielded findings consistent with acute non-specific pneumonitis. The patient received an additional antibiotic course of levofloxacin for 1 week without any improvement, and continued with daily febrile peaks. A thoracoscopy with open lung biopsy and wedge resection of the two largest infiltrates was performed, revealing confluent ill-defined granulomas mostly with purulent centres surrounded by an organizing pneumonia pattern, without evidence of vasculitis and with negative stains for infectious agents (Fig. 4).

In light of the finding of multiple granulomas on lung tissue, we considered an atypical pulmonary manifestation of Crohn's disease, and hence, treatment with non-selective infliximab was initiated. With this treatment the patient reported improved well-being with complete resolution of the fever, abdominal pain and diarrhoea. Four weeks later the pulmonary infiltrates on chest x-ray resolved completely. 


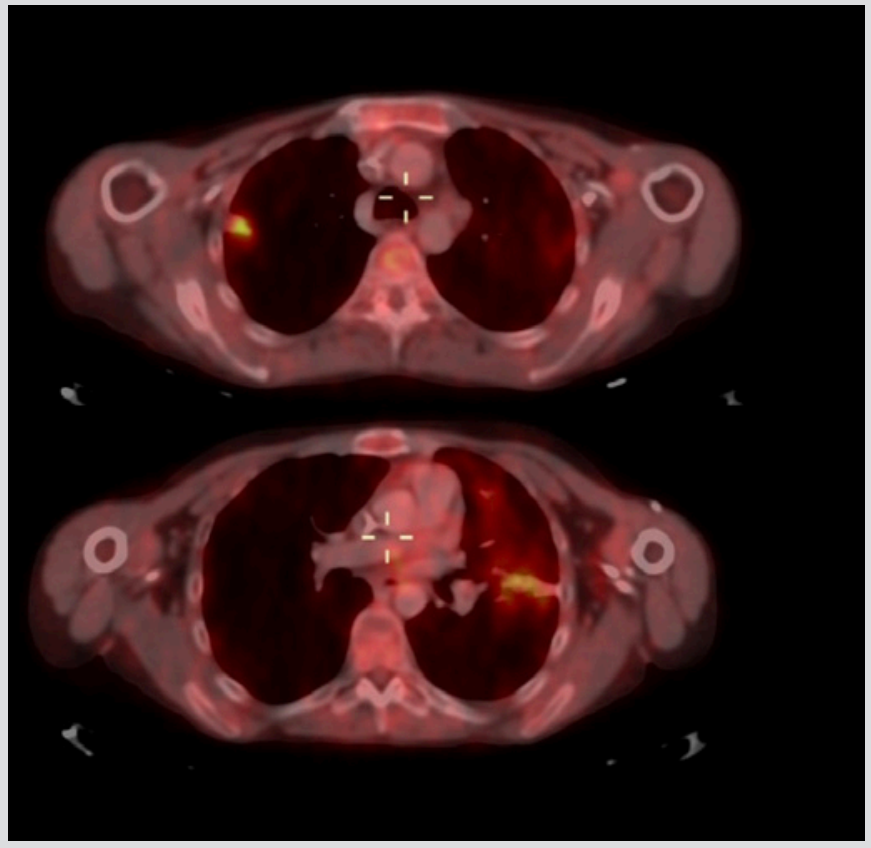

Figure 2. Top: PET-CT showing high intensity uptake in the periphery of the upper lobe of the right lung. Bottom: PET-CT showing similar uptake in the lower lobe of the left lung

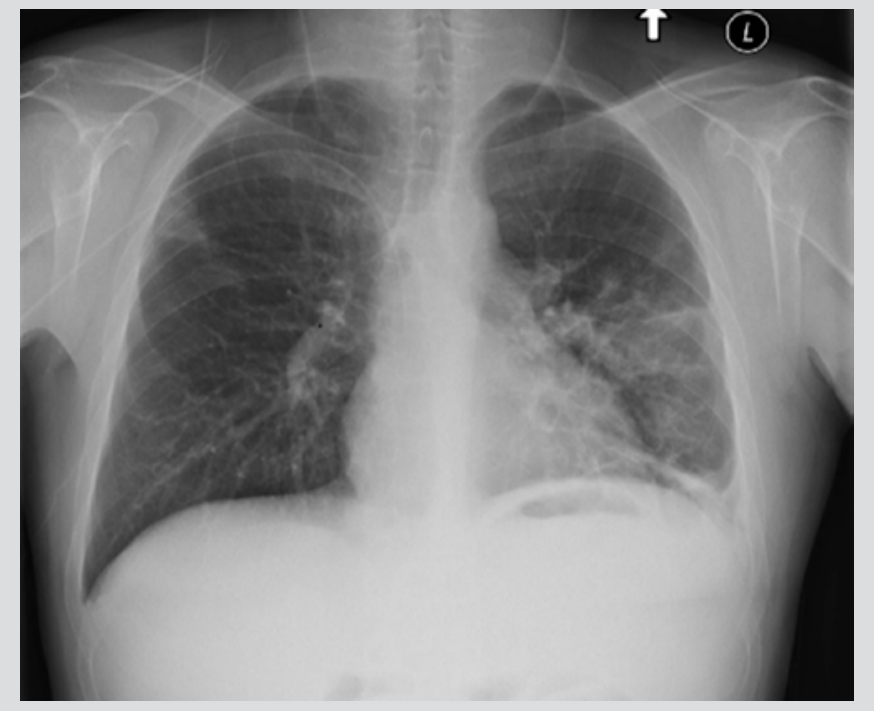

Figure 3. A chest $x$-ray revealing new bilateral infiltrates on both lungs and a new left pleural effusion

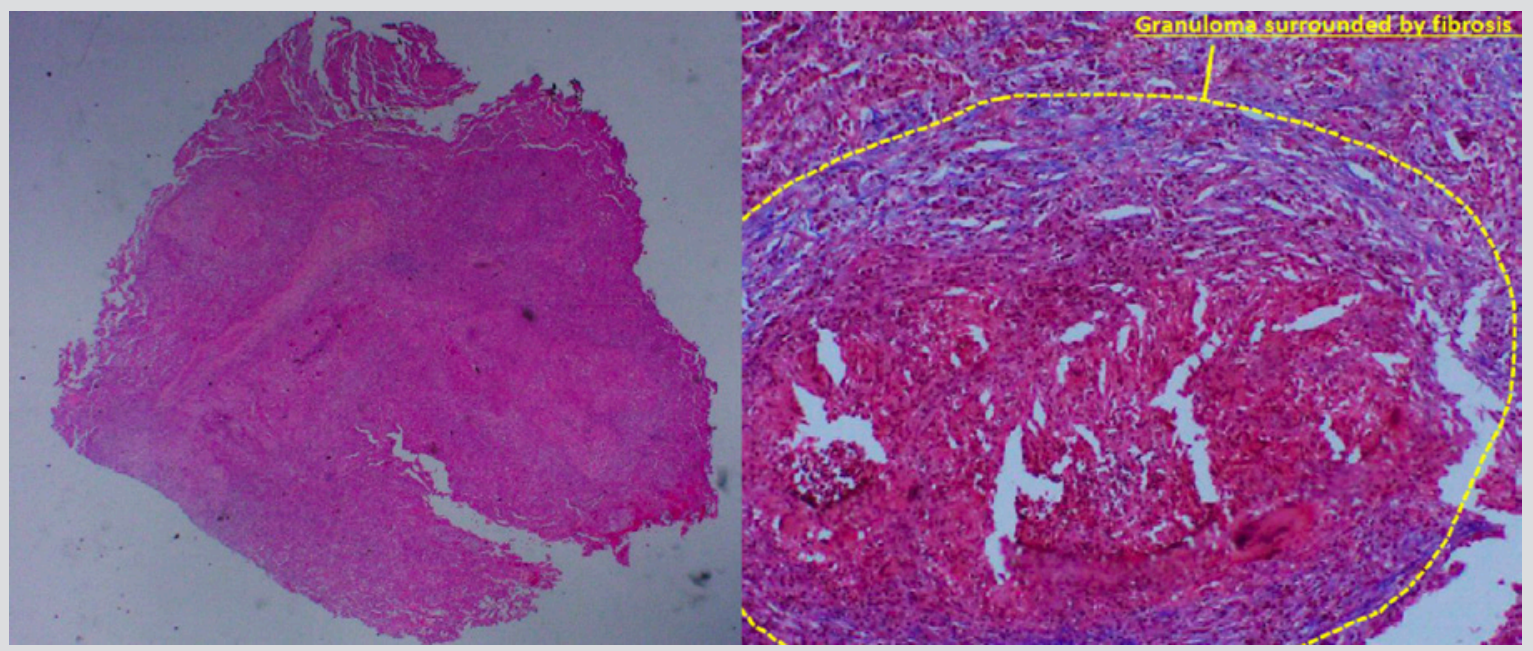

Figure 4.Left: lung parenchyma with many granulomas (H\&E 20x). Right: granuloma surrounded by fibrosis (Masson's trichrome 100x)

\section{DISCUSSION}

Crohn's disease is associated with extra-intestinal manifestations mainly involving the skin, joints, eyes and the hepatobiliary system. Less commonly, it can involve blood vessels, kidneys and the respiratory system.

Pulmonary involvement is relatively rare; however, there is a wide array of manifestations ranging from airway disease and pleural disease to lung parenchymal disease and drug-related diseases. The most frequently occurring of these is bronchial inflammation and suppuration of the upper airways with or without bronchiectasis ${ }^{[1]}$.There are multiple probable mechanisms by which the lung is injured; there is large similarity in the immune systems of both the pulmonary and intestinal mucosa ${ }^{[2]}$. In addition, there is evidence of circulating immune complexes and autoantibodies in patients with Crohn's disease that could lead to pulmonary injury ${ }^{[3]}$. 
Several forms of lung parenchymal involvement in Crohn's disease are recognized, including interstitial lung diseases such as cryptogenic organizing pneumonia, unspecified interstitial lung disease, non-caseating granulomatous inflammation and fibrosis ${ }^{[1]}$.

Chew et al. described a case of a pulmonary nodule that was biopsied and was found to contain both necrotizing and non-necrotizing granulomas; further work-up led to the diagnosis of colonic Crohn's disease, and treatment with infliximab led to resolution of both pulmonary and GI symptoms ${ }^{[4]}$.

Another case was described by Lissner et al. ${ }^{[5]}$ of a 28 -year-old patient assumed to have ulcerative colitis at presentation, who had received 3 infusions of vedolizumab, presenting with respiratory complaints and bilateral infiltrates on a chest CT scan. An open lung biopsy revealed non-caseating granulomas, while a repeat colonoscopy revealed similar granulomas along the Gl tract, leading to reclassification of the diagnosis to Crohn's disease with atypical pulmonary disease under vedolizumab treatment.

Vedolizumab is a monoclonal antibody that reduces chronically inflamed GI parenchymal tissue associated with ulcerative colitis and Crohn's disease by binding specifically to the $\alpha 4 \beta 7$ integrin receptor and blocking its interaction with mucosal addressin cell adhesion molecule 1. This inhibits the movement of memory T lymphocytes across the endothelium into inflamed GI tissue.

Lissner et al. analyzed integrin expression in whole blood samples from patients, showing neutralization of $\alpha 4 \beta 7$ on naive and memory $T$ lymphocytes as expected with vedolizumab treatment; interestingly, they observed a simultaneous increase in other integrins such as $\beta 1$ integrin in the lungs that led to altered migrational behaviour of leucocytes into organs other than the gut ${ }^{[5]}$.

Our case highlights some important points. First, pulmonary parenchymal manifestations are rare in Crohn's disease. They may be subclinical and difficult to diagnose. Our patient's chest x-ray was completely normal multiple times during his hospitalization, and he had no pulmonary-specific complaints until a few weeks into his illness. Pulmonary granulomas are an even rarer manifestation among Crohn's disease patients but they can be life-threatening if not diagnosed promptly.

We cannot be certain that there is a direct connection between treatment with vedolizumab and the emergence of the lung consolidations, although the chronological order of events strongly suggests this possibility. The resolution of GI symptoms and the evolution of respiratory disease occurred directly after treatment with vedolizumab, similar to the case presented by Lissner et al. ${ }^{[5]}$ described earlier.

We hypothesize that gut-selective treatments such as vedolizumab could unmask subclinical pulmonary involvement. The fact that stopping vedolizumab and replacing it with infliximab (anti-TNF) led to complete clinical and radiological resolution also supports this theory.

\section{REFERENCES}

1. Lu DG, Ji XQ, Liu X, Li HJ, Zhang CQ. Pulmonary manifestations of Crohn's disease. World J Gastroenterol 2014;20(1):133-141.

2. MacDermott RP, Nash GS, Nahm MH. Antibody secretion by human intestinal mononuclear cells from normal controls and inflammatory bowel disease patients. Immunol Invest 1989;18(1-4):449-457.

3. Bienenstock J, McDermott M, Befus D, O’Neill M. A common mucosal immunologic system involving the bronchus, breast and bowel. Adv Exp Med Biol 1978;107:53-59.

4. Chew MT, Chak E, Matsukuma K. A rare cause of pulmonary nodules. Case Rep Gastroenterol 2016;10(3):633-639.

5. Lissner D, Glauben R, Allers K, Sonnenberg E, Loddenkemper C, Schneider T, et al. Pulmonary manifestation of Crohn's disease developed under treatment with vedolizumab. Am J Gastroenterol 2018;113(1):146-148. 